\title{
VALENCY ENUMERATION OF ROOTED PLANE TREES
}

\author{
C. L. MALLOWS and $\mathrm{K}$. W. WACHTER \\ (Received 27 February 1970; revised 11 August 1970) \\ Communicated by G. Szekeres
}

\begin{abstract}
We obtain the enumerator by node-valencies of planted plane trees, whose square gives the enumerator of rooted plane trees. We also study the enumeration by number of nodes and black-node-valencies of bichromatic rooted plane trees, encountering a remarkably simple inversion formula. Finally, we remark that these bichromatic trees are in $1-1$ correspondence with solutions to the weak lead ballot problem.
\end{abstract}

\section{1}

A rooted tree is a tree with a single preferred node. A plane tree is a tree imbedded in the Euclidean plane and inheriting from the plane a sense of clockwise orientation; thus at each node except the root node there is exactly one branch leading to a node nearer to the root node, and at the root node and all other nodes except terminals there are one or more ordered branches leading to nodes further from the root node. Two such trees are isomorphic if their nodes can be placed in $1-1$ correspondence so as to preserve all adjacency and ordering relationships. Let $N\left(x_{1}, x_{2}, \cdots\right)$ be the enumerator of non-isomorphic rooted plane trees by node-valency; thus

$$
N(x)=x_{1}^{2}+2 x_{1}^{2} x_{2}+2 x_{1}^{3} x_{3}+3 x_{1}^{2} x_{2}^{2}+2 x_{1}^{4} x_{4}+8 x_{1}^{3} x_{2} x_{3}+4 x_{1}^{2} x_{2}^{3}+\cdots
$$

A shrub, also called a planted tree, is a rooted tree in which the root is of unit valency. To obtain an expression for $N$ it is convenient to discuss first the enumeration by valency of bichromatic plane shrubs, i.e. plane shrubs in which the root is colored black, while all other nodes are colored black or white in such a way that adjacent nodes are always colored oppositely. The five 4-node bichromatic rooted plane trees appear in a diagram at the end of this paper. The first two of them are shrubs.

Let $b_{1} M(\boldsymbol{b}, \boldsymbol{w})=b_{1} M\left(b_{1}, b_{2}, \cdots ; w_{1}, w_{2}, \cdots\right)$ be the enumerator of these shrubs, where the appearance of a factor $b_{k}$ in a term denotes the existence of a $k$ valent black node, and similarly for the $w^{\prime}$ s. We have 


$$
\begin{aligned}
b_{1} M(\boldsymbol{b}, \boldsymbol{w})= & b_{1} w_{1}+b_{1}^{2} w_{2}+b_{1}^{3} w_{3}+b_{1} b_{2} w_{1} w_{2}+b_{1}^{4} w_{4}+b_{1} b_{3} w_{1}^{2} w_{2} \\
& +2 b_{1}^{2} b_{2} w_{1} w_{3}+b_{1}^{2} b_{2} w_{2}^{2}+\cdots
\end{aligned}
$$

Enumeration by valency of the lowest white node leads immediately to the key result

$$
M(\boldsymbol{b}, \boldsymbol{w})=w_{1}+w_{2} M(\boldsymbol{w}, \boldsymbol{b})+w_{3} M^{2}(\boldsymbol{w}, \boldsymbol{b})+\cdots
$$

On dropping the color distinction we find

$$
x_{1}=\left(1-x_{2}\right) M(\boldsymbol{x}, \boldsymbol{x})-x_{3} M^{2}(\boldsymbol{x}, \boldsymbol{x})-\cdots
$$

In the sequel we use repeatedly the following result, which can be found in [1] (page 61) and follows simply from Lagrange's formula for the inversion of series (see [6] section 7.32).

LEMMA. If

then

$$
y=f_{1} x+f_{2} x^{2}+f_{3} x^{3}+\cdots
$$

$$
f_{1}^{r} x^{r}=r y^{r} \sum\left(-\frac{y f_{2}}{f_{1}^{2}}\right)^{a_{1}}\left(-\frac{y^{2} f_{3}}{f_{1}^{3}}\right)^{a_{2}} \cdots \frac{\left(r-1+2 a_{1}+3 a_{2}+\cdots\right) !}{\left(r+a_{1}+2 a_{2}+\cdots\right) ! a_{1} ! a_{2} ! \cdots}
$$

where the sum is over all nonnegative integers $a_{1}, a_{2}, \cdots$.

Applying this Lemma with $r=1$ and expanding negative powers of $\left(1-x_{2}\right)$ by the binomial theorem, we have from (2)

$$
M(\boldsymbol{x}, \boldsymbol{x})=\sum x_{1}^{t} x_{2}^{a_{2}} x_{3}^{a_{3}} \cdots\left(a_{2}+2 a_{3}+3 a_{4} \cdots\right) ! / t ! a_{2} ! a_{3} ! \cdots
$$

where $t=1+a_{3}+2 a_{4}+3 a_{5}+\cdots$ and the sum is over all nonnegative integers $a_{2} a_{3}, \cdots$. This result is equivalent to equation (8) in [5].

For bichromatic rooted plane trees, enumerating according to the valency of the root node, we have

$$
N(\boldsymbol{b}, \boldsymbol{w})=b_{1} M(\boldsymbol{b}, \boldsymbol{w})+b_{2} M^{2}(\boldsymbol{b}, \boldsymbol{w})+\cdots=M(\boldsymbol{b}, \boldsymbol{w}) M(\boldsymbol{w}, \boldsymbol{b}) .
$$

Dropping the color distinction and using the Lemma with $r=2$, we find

$$
N(\boldsymbol{x})=N(\boldsymbol{x}, \boldsymbol{x})=2 \sum x_{1}^{t+1} x_{2}^{a_{2}} x_{3}^{a_{3}} \cdots\left(1+a_{2}+2 a_{3}+\cdots\right) ! /(t+1) ! a_{2} ! a_{3} ! \cdots
$$

where $t=1+a_{3}+2 a_{4}+\cdots$ as before.

In the problem which led to these investigations [2] the main interest lay in the enumerator $N(y \boldsymbol{b}, y)$ (obtained from $N(\boldsymbol{b}, \boldsymbol{w})$ by replacing $b_{k}, w_{k}$ by $y b_{k}, y$ respectively, $k=1,2, \cdots)$ in which white nodes are merely counted and not enumerated by valency. From (1) we have on the one hand 
(4)

$$
M(y \boldsymbol{b}, y)=y /(1-M(y, y b))
$$

and on the other

$$
M(y, y \boldsymbol{b})=y b_{1}+y b_{2} M(y \boldsymbol{b}, y)+y b_{3} M^{2}(y \boldsymbol{b}, y)+\cdots
$$

so that $M(y b, y)$ satisfies

$$
M=y\left(1+b_{1} M+b_{2} M^{2}+\cdots\right)
$$

Also from (3) and (4) we find

$$
N(y \boldsymbol{b}, y)=M(y \boldsymbol{b}, \boldsymbol{y})-y .
$$

Solving (5) (by the Lemma) gives

$$
M(y \boldsymbol{b}, y)=\sum y^{n} b_{1}^{a_{1}} b_{2}^{a_{2}} \cdots\left(a_{1}+2 a_{2}+3 a_{3}+\cdots\right) ! /\left(n-a_{1}-a_{2} \cdots\right) ! a_{1} ! a_{2} ! \cdot
$$

where $n=1+a_{1}+2 a_{2}+\cdots$, and the sum is over all non-negative $a_{1}, a_{2}, \cdots$ Explicitly,

$$
\begin{aligned}
M(y \boldsymbol{b}, y)= & y+y^{2} b_{1}+y^{3}\left(b_{2}+b_{1}^{2}\right)+y^{4}\left(b_{3}+3 b_{1} b_{2}+b_{1}^{3}\right) \\
& +y^{5}\left(b_{4}+4 b_{3} b_{1}+2 b_{2}^{2}+6 b_{2} b_{1}^{2}+b_{1}^{4}\right)+\cdots
\end{aligned}
$$

\section{4}

Let us define $F(y, \boldsymbol{b}), \boldsymbol{f}(\boldsymbol{b})=\left\{f_{1}(\boldsymbol{b}), f_{2}(\boldsymbol{b}), \cdots\right\}$ by

$$
F(y, \boldsymbol{b})=y^{-1} M(y \boldsymbol{b}, y)=1+y f_{1}(\boldsymbol{b})+y^{2} f_{2}(\boldsymbol{b})+\cdots
$$

Notice that from (5) the function $F(y, b)$ satisfies

$$
F=1+y b_{1} F+y^{2} b_{2} F^{2}+\cdots
$$

Then the following remarkably simple result holds.

LemMA. If $F(y, b)$ satisfies (7) and is expanded as in (6), and if $G(\theta, x)=$ $1+\theta g_{1}(\boldsymbol{x})+\theta^{2} g_{2}(\boldsymbol{x})+\cdots$ satisfies

$$
G=1+\frac{\theta x_{1}}{G}+\frac{\theta^{2} x_{2}}{G^{2}}+\cdots
$$

then $G(\theta, f(b)) \equiv 1+\theta b_{1}+\theta^{2} b_{2}+\cdots$

Proof. By definition $G(\theta, f(b))$ satisfies

$$
G=1+\frac{\theta f_{1}(b)}{G}+\frac{\theta^{2} f_{2}(b)}{G^{2}}+\cdots
$$

so that according to (6)

$$
G(\theta, f(b))=F\left(\frac{\theta}{G}, \boldsymbol{b}\right)
$$


Now using (6) we find that $G(\theta, f(b))$ satisfies

$$
G=1+\frac{\theta}{G} b_{1} G+\left(\frac{\theta}{G}\right)^{2} b_{2} G^{2}+\cdots=1+\theta b_{1}+\theta^{2} b_{2}+\cdots
$$

as required.

Notice that we have also $F(y, g(z))=1+y z_{1}+y^{2} z_{2}+\cdots$, so that $F, G$ are in a sense inverses of one another. Explicitly,

$$
\begin{aligned}
& g_{1}(\boldsymbol{x})=x_{1} \\
& g_{2}(\boldsymbol{x})=x_{2}-x_{1}^{2} \\
& g_{3}(\boldsymbol{x})=x_{3}-3 x_{1} x_{2}+2 x_{1}^{3} \\
& g_{4}(\boldsymbol{x})=x_{4}-4 x_{1} x_{3}-2 x_{2}^{2}+10 x_{1}^{2} x_{2}-5 x_{1}^{4} .
\end{aligned}
$$

Solving (8) (using the Lemma with $r=-1$ ) we find

$$
\begin{array}{r}
G(\theta, \boldsymbol{x})=1-\sum\left(-\theta x_{1}\right)^{a_{1}}\left(-\theta^{2} x_{2}\right)^{a_{2}} \cdots\left(2 a_{1}+3 a_{2}+\cdots-2\right) ! / \\
a_{1} ! a_{2} !\left(a_{1}+2 a_{2}+\cdots-1\right) !
\end{array}
$$

where the summation is over all nonnegative $a_{1}, a_{2}, \cdots$ such that $a_{1}+a_{2}+\cdots \geqq 1$. This result was needed in [2].

If we write

$$
f_{n}(x, x, \cdots)=\sum f_{n, k} x^{k}
$$

then $f_{n, k}$ is the number of weak lead lattice paths from $(0,0)$ to $(n, n)$ having exactly $k$ horizontal (and $k$ vertical) segments, i.e., it is the number of solutions to the weak lead ballot problem that have $k$ blocks of votes for each candidate and finish with a $n-n$ tie. Such a lattice path can be described uniquely by the sequence $c_{1}, c_{2}, \cdots, c_{n}$ where $c_{1}=1, c_{i-1} \leqq c_{i} \leqq i ; c_{i}-1$ is the number of votes that have been accumulated by the trailing candidate $(B)$ when the leader's ( $A$ 's) $i$-th vote is counted. Explicitly,

$$
f_{n, k}=\frac{1}{n}\left(\begin{array}{l}
n \\
k
\end{array}\right)\left(\begin{array}{c}
n \\
k-1
\end{array}\right) \text {. }
$$

It is perhaps of interest to remark that there are two ways in which $c$ sequences can be placed in $1-1$ correspondence with $n+1$ node rooted plane trees:

(i) The first such correspondence is well known; one circumnavigates the tree, starting at the root, going up a new branch for each A-vote and down the other side of an old branch for each $B$-vote. This gives a vote-sequence and hence a c-sequence.

(ii) For another correspondence, color the nodes of the tree black and white alternately. Circumnavigate the tree, starting at the (black) root, assigning labels $1,2, \cdots, n$ to the black nodes as they are encountered. (We do not assign a label 
$n+1$ to the root node on completing the circuit.) Then for each black node, each of its labels is replaced by a copy of its lowest numerical label. This scheme results in the assignment of a total of $n$ labels to the black nodes. The set of assigned labels can then be reordered to form a c-sequence. And conversely, uniquely. For the five rooted plane trees with four nodes, the correspondences are shown in the diagram.

(i) vote-sequence $c$-sequence

(ii) Labels $c$-sequence vote-sequence

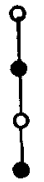

$a a a b b b$

111

122

122 $a b a a b b$

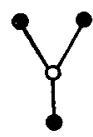

$a a b a b b$ 112

123

123

$a b a b a b$

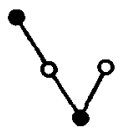

$a a b b a b$ 113

121

112

$a a b a b b$
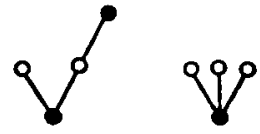

$a b a a b b$ $a b a b a b$ 123 122

113

111

113

111

$a a b b a b \quad a a a b b b$

Tutte [5] and Riordan [3], [4] have recently discussed some problems closely related to those considered in this paper.

We thank John Riordan for his interest in this problem and for his help in revising this paper; a referee's comments were also very helpful.

\section{References}

[1] F. N. David, M. G. Kendall and D. E. Barton, Symmetric Functions and Allied Tables (Cambridge University Press, 1966.)

[2] C. L. Mallows and K. W. Wachter, 'The asymptotic configuration of Wishart eigenvalues', in preparation.

[3] J. Riordan, 'Ballots and trees', J. Comb. Theory 6 (1969), 408-411.

[4] J. Riordan, 'Ballots and plane trees', unpublished manuscript.

[5] W. T. Tutte, 'The number of planted plane trees with a given partition'. Amer. Math. Monthly 71 (1964), 272-277.

[6] E. T. Whittaker and G. N. Watson, Modern Analysis (4th ed.) (Cambridge University Press, 1940).

Bell Telephone Laboratories, Incorporated

Murray Hill, New Jersey 